\title{
The burden of respiratory syncytial virus (RSV) associated acute lower respiratory infections in children with Down syndrome: A systematic review and meta-analysis
}

\author{
Markus Chan ${ }^{1 *}$, John J Park²*, \\ Ting $\mathrm{Shi}^{3}$, Federico Martinón- \\ Torres $^{4}$, Louis Bont ${ }^{5}$, Harish \\ Nair $^{1}$; Respiratory Syncytial \\ Virus Network (ReSViNET) \\ ${ }^{1}$ Usher Institute of Population Health \\ Sciences and Informatics, University of \\ Edinburgh, UK \\ ${ }^{2}$ Harvard TH Chan School of Public \\ Health, Harvard University, Boston, \\ Massachusetts, USA \\ ${ }^{3}$ Department of Public Health and \\ Primary Care, Leiden University Medical \\ Centre, Leiden, the Netherlands \\ ${ }^{4}$ Translational Pediatrics and Infectious \\ Diseases, Pediatrics Department, \\ Hospital Clínico Universitario de \\ Santiago de Compostela, Santiago de \\ Compostela, Spain \\ ${ }^{5}$ Wilhelmina Children's Hospital, \\ University Medical Center Utrecht, \\ Utrecht, Netherlands \\ * Joint first authroship
}

\begin{abstract}
Background Acute lower respiratory tract infections (ALRIs) caused by respiratory syncytial virus (RSV) are a leading cause of hospitalization in infants. Numerous risk factors have been identified in the aetiology of severe RSV-associated ALRI necessitating hospitalisation, including prematurity and congenital heart disease. Down syndrome (DS), a common genetic disorder associated with congenital and dysmorphic features, has recently been identified as an independent risk factor for RSV-associated ALRI requiring hospitalisation; however, the disease burden of RSV-associated ALRI in this population has not yet been established. Similarly, the impact of DS as an independent risk factor has not yet been quantified. We aimed therefore to estimate the incidence of admissions in children with DS, and by comparing this with unaffected children, to quantify the risk of DS independent of other risk factors.
\end{abstract}

Methods A systematic review of the existing literature published between 1995 and March 1, 2017 was performed to quantify the incidence of hospitalisation due to RSV-associated ALRI in children with DS. Meta-analyses were performed on extracted data using STATA statistical software, and hospitalisation rates for children with and without DS under the age of 2 were calculated.

Findings 5 articles were ultimately deemed eligible for analyses. Analyses were limited to children under the age of 2 years. We calculated the hospitalisation rate for children with DS in this age group to be 117.6 per 1000 child-years (95\% CI 67.4-205.2), vs a rate of 15.2 per 1000 child-years (95\% CI 8.3-27.6) in unaffected children. This indicates DS contributes to a 6.8 (95\% CI 5.5-8.4) fold increase in the relative risk of hospitalisation for RSV-associated ALRI.

Interpretation Though limited by a small number of articles, this review found sufficient evidence to conclude DS was a significant independent risk factor for the development of severe RSV-associated ALRI requiring hospitalisation. Further studies are needed to define the impact of DS in conjunction with other comorbidities on the risk of severe RSV infection. Determining benefits of immunoprophylaxis or future vaccines against RSV in this at-risk population is warranted.

Respiratory syncytial virus (RSV) is one of the leading causes of acute lower respiratory tract infections (ALRIs) in young children ( $<5$ years) globally [1-3].

Almost every child will have been infected with RSV by the age of 2 years, and $40 \%$ of these will develop ALRIs. Of these, $0.5-2 \%$ of children will require hos- 
pitalisation, making RSV-associated ALRIs the leading cause of hospitalisation among infants during winter [1]. Prematurity, chronic lung disease, age $<6$ months, and congenital heart disease (CHD) are established risk factors for RSV infection [4-6]. Bloemers et al. highlighted Down syndrome (DS) as an independent risk factor for RSV infection [7].

DS is the single most common chromosomal abnormality among live born infants, characterised by congenital and dysmorphic features [8]. Individuals with DS are more prone to numerous health issues, and the condition is associated with an overall increased risk of mortality $[9,10]$. Notably, ALRIs are the most common cause of hospitalisation among children with DS [11]. However, the burden of RSV-associated ALRI in children with DS is unclear. Further investigation into the epidemiology of RSV infection in paediatric DS populations may help optimise patient care in this vulnerable group.

We aimed to estimate the disease burden of RSV-associated ALRI in children with DS, focussing on hospitalisation and severity of disease. We also aimed to quantify the effect of DS as a dependent and independent risk factor for hospitalisation with RSV-associated ALRI.

\section{METHODS}

\section{Search strategy and selection criteria}

We conducted a systematic literature review using a combination of search terms, hand searching of online journals, and scanning of reference lists of identified citations. Two authors (JP and MC) searched the following databases - MEDLINE, Embase, Global Health, CINAHL, LILACS, Web of Science, IndMED, WHOLIS, and SIGLE. Both extracted data independently.

We included primary, population-based studies published between 1995 and March 1, 2017 reporting hospitalisation rates of RSV-associated ALRI in populations with DS over at least one (1) RSV season. Articles not available in English, and articles using an unclear or inconsistent case definition were excluded. Studies satisfying these minimum eligibility criteria were included. The full eligibility criteria are summarised in Table 1 .

The review was also registered on the PROSPERO database (Record \#59594). Full details of the search strategy used may be found on the PROSPERO database, and in Online Supplementary Document.

\section{Definitions}

ALRI has been defined as equivalent to clinical pneumonia, which is characterised by acute-onset cough or respiratory distress with age-adjusted tachypnoea. Down syndrome was defined as equivalent to having trisomy 21 based on medical or census records. RSV-associated ALRI was defined as ALRI in a child who is RSV positive (either based on laboratory confirmation or relevant ICD codes in medical records).

To increase the data available for analysis, methodology for confirming RSV infection or coding were not used as eligibility criteria, with the understanding that this could limit the interpretation of the results. We similarly did not distinguish between first or subsequent hospitalisations.

Table 1. Inclusion and exclusion criteria

\begin{tabular}{ll}
\hline Inclusion criteria & $\begin{array}{l}\text { Primary population-based studies reporting incidence data for RSV ALRI requiring hospitalisation } \\
\text { Articles with a surveillance period of at least l year or I RSV season }\end{array}$ \\
\hline Articles reporting case fatality ratios of RSV infection \\
\hline Articles assessing the OR or RR for children with DS for at least l year \\
\hline Articles confirming RSV infection by laboratory diagnosis or hospital discharge records of confirmed RSV \\
\hline Articles reporting estimates for children separately by immunoprophylaxis status, if included in the study \\
\hline All non-primary articles, including reviews, presentations \\
\hline Articles not available in English \\
\hline Articles not investigating RSV infection as a primary outcome \\
\hline Articles using a case definition of influenza or influenza-like illness \\
\hline Articles using an unclear or inconsistent case definition
\end{tabular}

RSV - respiratory syncytial virus, ALRI - acute lower respiratory tract infection, OR - odds ratio, RR - relative risk, DS - Down syndrome 


\section{Data extraction}

Hospitalisation rates for all populations and subgroups from relevant studies were extracted onto a Microsoft Excel 2013 database. Information regarding study characteristics, diagnostic tests used, and population characteristics was recorded. Outcome data and risk factor effect parameters were extracted if present. Relative risk and hospitalisation rate data were calculated using raw input data as well as reported figures in the reviewed studies.

\section{Data analysis}

We conducted meta-analyses of the data using STATA version 11.2 (StataCorp LP, College Station TX, USA). Primary outcomes were hospitalisation rates of RSV-associated ALRI in children with DS or without DS, and the impact of DS on hospitalisation rate expressed as relative risk. Pooled estimates with corresponding 95\% CIs were reported based on random effects model (DerSimonian-Laird method) since significant heterogeneity was expected across studies [12,13].

Two meta-analyses were performed: one using hospitalisation rate data, and one with relative risk data. Sensitivity analyses by age were planned, but were not performed due to limited data.

\section{RESULTS}

The literature search identified 111 records for screening after excluding duplicates. Of these, 50 articles were reviewed fully, and 5 articles were ultimately identified as meeting our strict eligibility criteria $[7,14-$ 17]. This is shown in Figure 1.

All 5 studies used passive hospital data and 1 used both active and passive methods of data collection. Study populations were under the age of 2 years $[7,14,15,17]$, except one study which included infants up to 36 months [16]. All studies used hospital in-patient or out-patient data, and did not distinguish between first or repeat hospitalisation episodes. All studies were based in high-income countries: 3 studies were from Europe and 2 were from the USA. No studies in Asia, Africa or South America met eligibility criteria. These articles are summarised in Table $2[7,14-17]$.

Data analyses focused on the $<2$ years old age group, given the paucity of data beyond this group. The implications of this decision are discussed below.

\section{Hospitalisation rate}

Four of the 5 studies were included in the meta-analysis of hospitalisation rates for children under the age of 2 years with and without DS. The meta-analysis found the hospitalisation rate of RSV-associated ALRI in children under the age of 2 years with DS was 117.6 (95\% CI 67.5-205.2) per 1000 children

152 records identified through database search

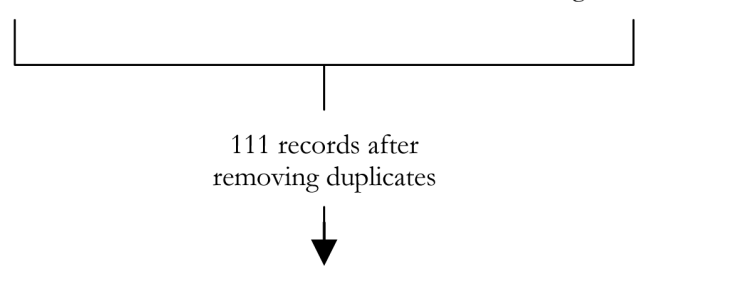

111 records screened

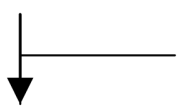

61 records excluded for irrelevance

50 full-text articles assessed for eligibility

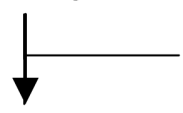

45 articles excluded by eligibility criteria

5 full-text articles retained for analyses

Figure 1. PRISMA flow diagram. per year. Comparatively, the hospitalisation rate of RSV-associated ALRI in children under the age of 2 years without DS was 15.2 (95\% CI 8.3-27.6) per 1000 children per year, $(P<0.01)$.

The meta-estimate of relative risk of DS for hospitalisation with RSV-associated ALRI (including data from all 5 studies) in this age group was 6.8 (95\% CI 5.5-8.4).

These findings are summarised in Table 3 and Figure 2 .

\section{Disease severity data}

Four of the 5 studies compared disease severity in children with and without DS; however, this was inconsistent. Distinct criteria were used across the studies, including need for respiratory support $[16,17]$, need for mechanical ventilation $[16,17]$, median duration of hospital stay $[14,16,17]$, and mean duration of stay [15]. 
Table 2. Summary of articles in review

\begin{tabular}{|c|c|c|c|c|c|c|}
\hline Authors & Study setting & StUdY PERIOD & Population & $\begin{array}{l}\text { HospitaLISATION RATE } \\
\text { (PER } 1000 \text { CHILD-YEARS) }\end{array}$ & $\begin{array}{c}\text { OR/RR OF } \\
\text { HOSPITALISATION } \\
\text { (DS vS NON-DS) }\end{array}$ & Severity MEASUREMENT \\
\hline \multirow{2}{*}{$\begin{array}{l}\text { Bloemers et al. } \\
\text { [7] }\end{array}$} & \multirow{2}{*}{$\begin{array}{l}\text { Amsterdam } \\
\text { and Leiden, } \\
\text { Netherlands }\end{array}$} & \multirow[t]{2}{*}{$1976-2005$} & \multirow{2}{*}{$\begin{array}{c}<36 \text { mo; } 395 \text { DS; } 276 \\
\text { non-DS; Not receiving } \\
\text { palivizumab }\end{array}$} & DS: 98.7 (72.1-135.1) & \multirow{2}{*}{$\begin{array}{c}13.6 \\
(3.3-56.4)\end{array}$} & \\
\hline & & & & Non-DS: 7.3 (1.8-29.0) & & \\
\hline \multirow{2}{*}{$\begin{array}{l}\text { Fjaerli et al. } \\
\text { [14] }\end{array}$} & \multirow{2}{*}{$\begin{array}{l}\text { Akershus, } \\
\text { Norway }\end{array}$} & \multirow[t]{2}{*}{$1993-2000$} & \multirow{2}{*}{$\begin{array}{c}<2 \text { y; } 7 \text { DS; } 70 \text { other } \\
\text { non-DS high-risk; } 687 \\
\text { non-DS; Low-risk }\end{array}$} & DS: 153.9 (76.9-307.6) & \multirow{2}{*}{$\begin{array}{c}11.0 \\
(5.5-22.0)\end{array}$} & \multirow{2}{*}{$\begin{array}{l}\text { Median LoS; DS: } 7.5 \mathrm{~d} ; \\
\text { Non-DS: } 4 \mathrm{~d} ;(P<0.001)\end{array}$} \\
\hline & & & & Non-DS: $14.0(13.1-15.0)$ & & \\
\hline \multirow{2}{*}{$\begin{array}{l}\text { Kristensen et al. } \\
{[15]}\end{array}$} & \multirow[t]{2}{*}{ Denmark } & \multirow[t]{2}{*}{$1997-2003$} & \multirow{2}{*}{$\begin{array}{c}\text { O-23 mo;399 DS; } \\
391584 \text { non-DS }\end{array}$} & DS: 195.5 (156.6-244.0) & \multirow[t]{2}{*}{$7.1(5.7-8.9)$} & \multirow{2}{*}{$\begin{array}{c}\text { Mean LoS; DS: } 1.91 \mathrm{~d} ; \\
\text { Non-DS: } 1.0 \mathrm{~d} \\
(P<0.001) \\
\end{array}$} \\
\hline & & & & Non-DS: 27.5 (27.0-28.0) & & \\
\hline \multirow{2}{*}{$\begin{array}{l}\text { Stagliano et al. } \\
\text { [16] }\end{array}$} & \multirow[t]{2}{*}{ USA } & \multirow[t]{2}{*}{$2005-2011$} & \multirow{2}{*}{$\begin{array}{l}<36 \text { mo; } 842 \text { DS; } \\
632358 \text { non-DS }\end{array}$} & DS: $96.2(77.4-119.6)$ & \multirow[t]{2}{*}{$6.8(5.5-8.4)$} & \multirow{2}{*}{$\begin{array}{l}\text { Need for respiratory support; } \\
\text { DS: 9.3\%; Non-DS: } 1.8 \% \text {; } \\
(P<0.001) \text {; Median LoS; DS: } \\
4 \text { d; Non-DS: } 2 \text { d; }(P<0.01)\end{array}$} \\
\hline & & & & Non-DS: 14.2 (13.9-14.5) & & \\
\hline \multirow{2}{*}{$\begin{array}{l}\text { Zachariah et al. } \\
\text { [17] }\end{array}$} & \multirow{2}{*}{$\begin{array}{l}\text { Colorado, } \\
\text { USA }\end{array}$} & \multirow[t]{2}{*}{$1995-2006$} & \multirow{2}{*}{ o-23 mo; 630 DS; } & DS: 67.5 (49.9-91.1) & \multirow[t]{2}{*}{$6.0(5.4-6.7)$} & \multirow{2}{*}{$\begin{array}{c}\text { Median LoS; DS: 4-5 d; } \\
\text { Non-DS: } 2-3 \mathrm{~d} ;(P<0.001)\end{array}$} \\
\hline & & & & Non-DS: 12.1 (11.9-12.3) & & \\
\hline
\end{tabular}

DS - patients with Down syndrome, Non-DS - patients without Down syndrome, RR - relative risk, OR - odds ratio, LoS - length of stay, d - day, mo - month, y-year

Table 3. Summary of articles, with meta-analyses and sensitivity analyses

\begin{tabular}{|c|c|c|c|}
\hline Author & $\begin{array}{l}\text { RSV-ASSOciated ALRI HOSPITALISATION RATE AMONG } \\
\text { PATIENTS WITH DS, PER } 1000 \text { CHILD-YEARS (95\% CI) }\end{array}$ & $\begin{array}{l}\text { RSV-ASSOCIATED ALRI hOSPITALISATION RATE AMONG } \\
\text { PATIENTS WITHOUT DS, PER } 1000 \text { CHILD-YEARS (95\% CI) }\end{array}$ & RR (95\% CI) \\
\hline Bloemers et al. [7] & $98.7(72.1-135.1)$ & $7.3(1.8-29.0)$ & $13.6(3.3-56.4)$ \\
\hline Fjaerli et al. [14] & $153.9(76.9-307.6)$ & $14.0(13.1-15.0)$ & $11.0(5.5-22.0)$ \\
\hline Kristensen et al. [15] & $195.5(156.6-244.1)$ & $27.5(27.0-28.0)$ & $7.1(5.7-8.9)$ \\
\hline Stagliano et al. [16] & $96.2(77.4-119.6)$ & $14.2(13.9-14.5)$ & $6.8(5.5-8.4)$ \\
\hline Zachariah et al. [17] & $67.5(49.9-91.1)$ & $12.1(11.9-12.3)$ & $6.0(5.4-6.7)$ \\
\hline \multicolumn{4}{|l|}{ Meta-analysis of all articles: } \\
\hline Meta-analysis for all under 2 years* & $117.6(67.4-205.2)$ & $15.2(8.3-2.6)$ & $6.8(5.5-8.4)$ \\
\hline
\end{tabular}

RSV - respiratory syncytial virus, ALRI - acute lower respiratory tract infection, RR - relative risk, CI - confidence interval

* Sensitivity analysis using data from Bloemers et al. [7], Fjaerli et al. [14], Kristensen et al. [15], and Zachariah et al. [17] only.

The wide range of severity measures precluded formal analyses of the data; however, within the group of hospitalised children, RSV-associated ALRI was consistently more severe in children with DS compared to those without DS [14-17]. This is shown in Table 2.

\section{Comorbidities}

Three of the 5 studies investigated the role of comorbid risk factors in the epidemiology of RSV-associated ALRI in children with DS. However, there were inconsistencies in the conditions assessed, and the parameters used.

Only two studies formally assessed the impact of comorbid risk factors; however, analyses were limited to comparing populations with DS and any additional risk factors to populations with DS without any risk factors $[7,17]$. The inconsistencies between the studies precluded formal meta-analyses of the data. Both studies found the presence of additional risk factors generally did not significantly affect the hospitalisation rate, clinical presentation, or management of RSV-associated ALRI in children with DS [7,17]. However, Zachariah et al. noted a significantly higher use of bronchodilators in populations with DS and at least one additional risk factor compared to populations with DS alone [17].

Importantly, all three studies found an increased hospitalisation rate for RSV-associated ALRI in populations with DS without additional risk factors $[7,16,17]$.

\section{DISCUSSION}

To our knowledge, this is the first systematic review and meta-analysis to report global estimates for hospitalisation rates attributable to RSV-associated ALRI in children with DS compared to children without 

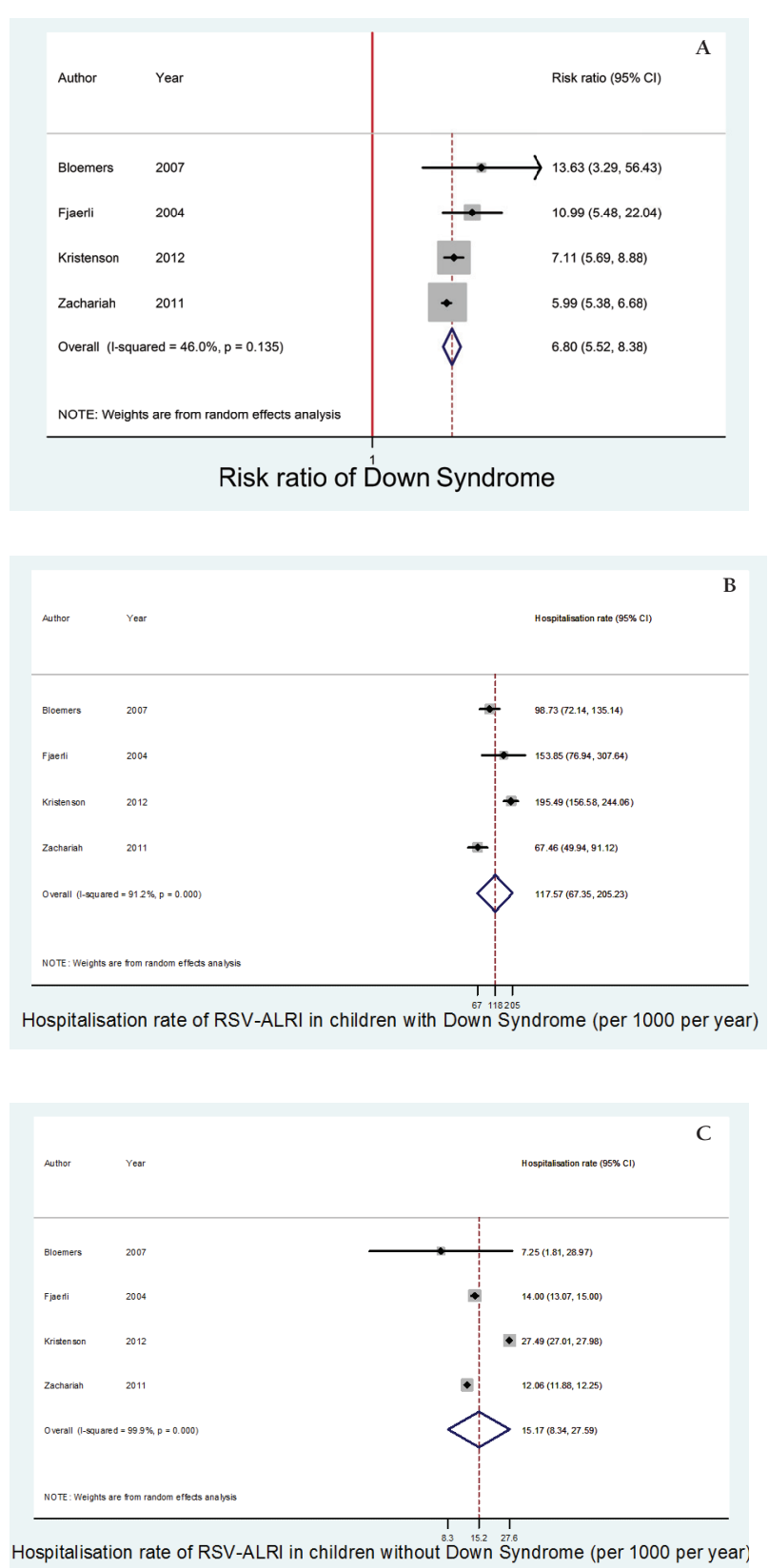

Figure 2. Forest plots of (a) risk ratio of Down syndrome for respiratory syncytial virus (RSV)-associated ALRI requiring hospitalisation in children under the age of 2 years; (b) hospitalisation rate of RSV-associated acute lower respiratory tract infection (ALRI) in children under the age of 2 years with Down syndrome; and (c) hospitalisation rate of RSVassociated ALRI in children under the age of 2 years without Down syndrome.
DS. This is important since RSV remains a leading cause of ALRIs and hospitalisation among children globally [1,2]. We estimated children under the age of 2 years with DS had a 6.8 times (95\% CI 5.5-8.4) increased risk of hospitalisation for RSV-associated ALRI compared to children in the same age group without DS. This suggests DS is an important risk factor for RSV-associated ALRI hospitalisation and comparable to other risk factors like CHD (RR 1.5-2.7) [18-21] and bronchopulmonary dysplasia (RR 10.7) [21].

Though meta-analyses were not performed on severity measures, studies assessing infection severity consistently found more severe RSV-associated ALRI in hospitalized children with DS compared to those without DS. Generally, a higher proportion of children with DS required respiratory support and required longer hospitalisation [14-17].

\section{Down syndrome and RSV infection}

Several proposed mechanisms explain the increased susceptibility of children with DS to RSV-associated ALRI. These children are more likely to have airway abnormalities such as poor upper airway tone and abnormal lung development. These may contribute towards a tendency to airway compromise and oedema, and thus severe ALRIs [15,22,23]. Children with DS have also been shown to have high rates of CHD and pulmonary hypertension, both of which are risk factors for severe RSV-associated ALRI $[8,20]$. While these comorbidities are independent risk factors for the development of severe RSV-associated ALRI, the impact of these risk factors in conjunction with DS remains unclear.

Immunological dysfunction may also have a role: DS has been associated with poor thymus development and lower numbers of $\mathrm{B}$ and $\mathrm{T}$ cells, which may contribute to prolonged infections and poor microbial clearance [24-27].

Lastly, the presence of DS may be associated with a lower threshold for admission, given the complex care needs which may be present in affected infants. This is particularly true in the first year of life, where there is generally poorer nutritional intake $[16,28]$. However, this does not fully explain the increased risk suffered by children with DS, and disease severity likely also has a role [7].

\section{Comorbidities and Down syndrome}

Only three studies reported hospitalisation rate data in populations with DS and other comorbidities, limiting formal analyses on the role of comorbid risk factors alongside DS in the epidemiology of RSV-associated ALRI [7,16,17].

Children with DS often have other risk factors for severe RSV-associated ALRI. Notably, CHD, a significant risk factor for severe RSV-associated ALRI, is comorbid in up to $55 \%$ of children with DS $[29,30]$. However, the reviewed studies consistently showed children with DS alone remained at significantly greater risk of severe RSV-associated ALRI necessitating hospitalisation $[7,16,17]$.

This review suggests there are limited studies exploring the impact of DS in conjunction with other risk factors in the epidemiology of RSV-associated ALRI. Our preliminary findings suggest children with DS and other risk factors are at similar risk of severe RSV-associated ALRI compared to children with DS alone, but further research is necessary [7,16,17]. 


\section{Palivizumab prophylaxis for children with Down syndrome}

Palivizumab is a monoclonal IgG-1 antibody which has been shown to reduce the incidence of RSV hospitalisation, particularly in high-risk groups [31]. However, due to the high costs associated with it, licensing has largely been limited to high-risk populations such as infants with haemodynamically significant CHD (HSCHD) [32].

This systematic review suggests DS alone is associated with a significantly increased risk of hospitalisation due to RSV-associated ALRI. Furthermore, our results suggest that DS alone is associated with more severe RSV infection. While a meta-analysis could not be performed, the consistency of the findings is striking. However, there are other factors to consider when discussing the benefit of immunoprophylaxis in this population.

There are currently no large randomised-control trials investigating the efficacy of immunoprophylaxis for the prevention of RSV-associated ALRI specifically in children with DS. Yi et al. found a lower rate of RSV-related hospitalisation in infants with DS who were treated with palivizumab compared to a retrospective group of untreated controls [33]. A recent observational study by Mikami et al. investigated the efficacy of palivizumab in preventing RSV-associated hospitalisations in immunocompromised populations, including children with DS. However, the study was uncontrolled, and data for children with DS were not explicitly available. Further studies are therefore required to determine the efficacy of palivizumab in patients with DS.

Currently, guidance for palivizumab variably includes a statement on DS. Where this is explicitly mentioned, the guidance further varies, from not routinely recommended to reasonably recommended $[33,34]$. Many groups cite uncertainty over the burden of disease and the efficacy of palivizumab immunoprophylaxis in this population as challenges limiting the guidance [33-35].

Despite being at increased risk, no studies investigating RSV vaccination or new immunoprophylactic agents to our knowledge have included children with DS in their study populations. Similarly, further information on the topic will be similarly needed to guide RSV prevention in children with DS. Notably, studies on safety and cost-effectiveness in this at-risk population would be helpful in establishing guidance on this issue.

\section{Study limitations}

The main limitation in this study was the small number of articles eligible for analysis. This reflects the paucity of research in this area, and demonstrates the need for further investigation into the epidemiology of RSV-associated ALRIs in populations with DS. Notably, several studies pooled populations with and without immunoprophylaxis, and were therefore excluded from the meta-analyses.

Similarly, data were limited regarding the role of comorbidities and other confounding factors such as CHD and immunodeficiency. Only three studies assessed the role of DS in addition to other risk factors. While all three studies found DS to be an independent risk factor for RSV-associated hospitalization, the role of DS in addition to other risk factors in the epidemiology of RSV-associated ALRI is likely complex and currently not clearly understood $[7,15]$.

In particular, the excess risk carried by DS in conjunction with major risk factors remains unknown, and clarification in this area would help define the vulnerability of this at-risk population.

Inconsistencies between the studies reviewed also limited the internal validity of this study. Due to limited data, analyses were restricted to children under the age of 2 years. While RSV-associated ALRI primarily affects younger infants ( $<2$ years), severe infection has also been reported in older children $(<5$ years) $[2,16]$. This may be especially true for children with DS: this review found children with DS were older at admission than control populations, though this was statistically insignificant $[14,16,17]$. However, at least one study has shown children with DS to be significantly older than unaffected children at time of hospitalisation [36].

Similarly, the reporting of comorbidities and disease severity, where available, was variable. Notably, studies assessing the impact of risk factors did so by the general presence or absence of risk factors, limiting comparisons.

The precise definition of RSV-associated ALRI varied between studies, though they were broadly similar. Differences arose primarily in case ascertainment, with some studies relying on laboratory-confirmed RSV status $[7,14]$ and others using hospital discharge coding [15-17]. Due to paucity of studies, we did not 
analyse data by method of case ascertainment. Notably, this review is thus subject to the limitations of the studies included, particularly regarding the accuracy of ICD coding.

Reporting overall hospitalisation rates may over-represent more vulnerable individuals, resulting in inaccurate data. This effect may be exaggerated when considering some guidelines advocate admitting children for social or geographic reasons [37,38]. Risk factors for repeated admission may be distinct from risk factors of severe infection, and these nuances may be lost when measuring hospitalisation rates alone.

A greater limitation in the application of this review is the scope of the review, and the socio-geographical bias of the eligible articles. Only five studies were retained for the meta-analyses, and all were based either in Europe or North America. Notably, no articles from low- and middle-income countries (LMICs) fulfilled eligibility criteria, though previous reviews have highlighted the significant disease burden of RSVassociated ALRI in such countries $[2,39]$. While DS may reasonably be assumed to be a risk factor everywhere, the burden of disease in these populations may vary depending on local public health programmes.

Another limitation of this review was the age of the studies included in the review. Much of the data used in the meta-analyses came from before 2005. As population health and the efficacy of medical interventions have improved in this time, and continue to do so, it is possible the data do not reflect current trends in RSV-associated ALRI hospitalisation and standards of hospital care; this is especially true in high income settings.

Guidelines for palivizumab immunoprophylaxis have also changed during this time, notably for children with HSCHD [33,40]. The treatment of HSCHD has similarly improved in recent years [41]. Theoretically, correcting HSCHD should reduce the risk of developing RSV-associated ALRI, though there are yet limited data to support this hypothesis. These developments could also affect the data in this review.

\section{Future research}

The meta-analysis identified a significant need for good data on the impact of RSV-associated ALRI in populations with DS. Notably, further research into the impact of DS on the epidemiology of RSV-associated ALRI in combination with other risk factors would help clarify the burden of disease in this population. Similarly, research investigating the risk carried by populations with DS over time could give insight into the pathophysiology underlying this relationship. This would also help define the risk of children with DS to develop repeat episodes of RSV-associated ALRI.

Importantly, all the studies included in this review came from high-income countries in Europe or the Americas. Data from other geographic regions, particularly in LMICs, would help identify regional variations, and may help establish guidance for RSV prevention globally.

Similarly, to assess better the role of immunoprophylaxis, studies investigating the efficacy and tolerability of palivizumab specifically in infants with DS are needed. Cost-effectiveness analyses in this population may also be helpful, though these analyses may be limited by geographic variation in the costs and benefit of immunoprophylaxis. Furthermore, as palivizumab immunoprophylaxis becomes introduced more widely, the effects of changing policies on the epidemiology of RSV-associated ALRI in this population should be explored.

\section{CONCLUSION}

This is the first systematic review investigating the burden of RSV-associated ALRI in children with Down syndrome. Our findings suggest this population is at significantly greater risk of severe RSV-associated ALRI than unaffected populations, and may benefit from being considered for immunoprophylaxis. Unfortunately, the efficacy of palivizumab in this population is still unclear, and warrants further research, including cost-effectiveness analysis. When vaccines or extended half-life antibodies against RSV will become available, their efficacy should be explored in this vulnerable population. 
Acknowledgment: We would like to acknowledge ReSViNET members for their support. The members of ReSViNET include: 1. Louis Bont, Diederick E Grobbee, University Medical Center Utrecht, Utrecht, The Netherlands. 2. Anne Greenough, Center for Vaccines and Immunity. Nationwide Children's Hospital, Columbus, Ohio, United States of America. 3. Harish Nair, Usher Institute of Population Health Sciences and Informatics, Edinburgh Medical School, The University of Edinburgh, Edinburgh, United Kingdom. 4. Paolo Manzoni, Neonatal Intensive Care Unit. S. Anna Hospital, Torino, Italy. 5. Nikolaos Papadopoulos, Department of Allergy, Second Pediatric Clinic, University of Athens, Greece. 6. Eugenio Baraldi, Women's and Children's Health Department, Unit of Respiratory Medicine and Allergy, Padova. Italy. 7. Ann R Falsey, University of Rochester School of Medicine, and Rochester General Hospital, Rochester, NY, USA. 8. Terho Heikkinen, Department of Paediatrics, University of Turku and Turku University Hospital, Turku, Finland. 9. Asunción Mejías, Center for Vaccines and Immunity. Nationwide Children's Hospital. Columbus. Ohio. United States of America. 10. Fernando P Polack, Division of Infectious Diseases. St. George's Healthcare NHS Trust. United Kingdom. 11. Mike Sharland, Division of Infectious Diseases. St. George's Healthcare NHS Trust. United Kingdom. 12. Octavio Ramilo, Center for Vaccines and Immunity. Nationwide Children's Hospital. Columbus. Ohio. United States of America. 13. Renato T Stein, Department of Pediatrics, School of Medicine, Pontifica Universidade Católica, Porto Alegre, Brazil. 14. Federico Martinón-Torres, Hospital Clínico Universitario de Santiago de Compostela, Santiago de Compostela, Spain, 15. Peter D Sly, Queensland Children's Medical Research Institute, Brisbane, Australia. 16. Diederick E Grobbee, Julius Clinical, Netherlands. 17. Marta Nunes, Respiratory and Meningeal Pathogens Research Unit, Medical Research Council, University of the Witwatersrand, South Africa.

Funding: None.

Authorship contributions: HN conceptualised the study and provided oversight. MC and JP conducted the literature review and data analysis and wrote the first draft of the manuscript. TS contributed to data analysis and report writing. HN, LB and FMT contributed to data interpretation and critically reviewed and revised the initial draft. LB, FMT and HN are members of the Respiratory Syncytial Virus Network (ReSViNET).

Competing interests: HN, LB and FMT are members of the Respiratory Syncytial Virus Consortium in Europe (RESCEU). RESCEU has received funding from the Innovative Medicines Initiative 2 Joint Undertaking under grant agreement No. 116019. This Joint Undertaking receives support from the European Union's Horizon 2020 research and innovation programme and EFPIA.

FM-T's institution has received both funding for investigator initiated studies from Astra, MSD and Pfizer and trials fees from Regeneron, Medimmune, Pfizer, MSD, Sanofi Pasteur, Novartis, GSK, Ablynx, Janssen and Novavax. FM-T has received speaker and/or consultancy honoraria from Pfizer, MSD, Sanofi Pasteur, Novartis and GSK. FM-T research activities have been supported by grants from Consellería de Sanidade, Xunta de Galicia (RHIO7/2-intensificación actividad investigadora, PS09749 and 1OPXIB918184PR), Instituto de Salud Carlos III (Intensificación de la actividad investigadora 2007-2017), Fondo de Investigación Sanitaria (FIS; PI070069/PI1000540/PI1601569) del plan nacional de I + D + I and 'fondos FEDER' and 2016-PG071 Consolidación e Estructuración REDES 2016GI-1344 G3VIP (Grupo Gallego de Genética Vacunas Infecciones y Pediatría, ED341D R2016/021). FM-T reports funding from Ablynx, Jansen, GSK, Regeneron, and Medimmune outside the submitted work.

MC, JJP and TS have nothing to disclose.

HN reports grants from Innovative Medicines Initiative, grants and personal fees from BMGF, grants from Sanofi Pasteur, personal fees and non-financial support from MedImmune, outside the submitted work. LB has regular interaction with pharmaceutical and other industrial partners. He has not received personal fees or other personal benefits. UMCU has received major funding (>€100000 per industrial partner) for investigator initiated studies from AbbVie, MedImmune, Janssen, the Bill and Melinda Gates Foundation and MeMed Diagnostics. UMCU has received minor funding participation in trials by Regeneron and Janssen since 2015 (total annual estimate less than $€ 20000$ ). He received minor funding for consultation and invited lectures by AbbVie, MedImmune, Ablynx, Bavaria Nordic, MabXience, Novavax, Janssen (total annual estimate less than $€ 20000)$. 
1 Glezen WP, Taber LH, Frank AL, Kasel JA. Risk of primary infection and reinfection with respiratory syncytial virus. Am J Dis Child. 1986;140:543-6. Medline:3706232

2 Nair H, Nokes DJ, Gessner BD, Dherani M, Madhi SA, Singleton RJ, et al. Global burden of acute lower respiratory infections due to respiratory syncytial virus in young children: a systematic review and meta-analysis. Lancet. 2010;375:154555. Medline:20399493 doi:10.1016/S0140-6736(10)60206-1

3 Rudan I, Boschi-Pinto C, Biloglav Z, Mulholland EK, Campbell H. Epidemiology and etiology of childhood pneumonia. Bull World Health Organ. 2008;86:408-16. Medline:18545744 doi:10.2471/BLT.07.048769

4 Figueras-Aloy J, Carbonell-Estrany X, Quero J; IRIS Study Group. Case-control study of the risk factors linked to respiratory syncytial virus infection requiring hospitalization in premature infants born at a gestational age of 33-35 weeks in Spain. Pediatr Infect Dis J. 2004;23:815-20. Medline:15361718 doi:10.1097/01.inf.0000136869.21397.6b

5 Hall CB, Kopelman AE, Douglas RG Jr, Geiman JM, Meagher MP. Neonatal respiratory syncytial virus infection. N Engl J Med. 1979;300:393-6. Medline:759915 doi:10.1056/NEJM197902223000803

6 MacDonald NE, Hall CB, Suffin SC, Alexson C, Harris PJ, Manning JA. Respiratory syncytial viral infection in infants with congenital heart disease. N Engl J Med. 1982;307:397-400. Medline:7088112 doi:10.1056/NEJM198208123070702

7 Bloemers BL, van Furth AM, Weijerman ME, Gemke RJ, Broers CJ, van den Ende K, et al. Down syndrome: a novel risk factor for respiratory syncytial virus bronchiolitis—a prospective birth-cohort study. Pediatrics. 2007;120:e1076-81. Medline:17908728 doi:10.1542/peds.2007-0788

8 Roizen NJ, Patterson D. Down's syndrome. Lancet. 2003;361:1281-9. Medline:12699967 doi:10.1016/S01406736(03)12987-X

9 Presson AP, Partyka G, Jensen KM, Devine OJ, Rasmussen SA, McCabe LL, et al. Current estimate of Down Syndrome population prevalence in the United States. J Pediatr. 2013;163:1163-8. Medline:23885965 doi:10.1016/j. jpeds.2013.06.013

10 Malt EA, Dahl RC, Haugsand TM, Ulvestad IH, Emilsen NM, Hansen B, et al. Health and disease in adults with Down syndrome. Tidsskr Nor Laegeforen. 2013;133:290-4. Medline:23381164 doi:10.4045/tidsskr.12.0390

11 Leonard S, Bower C, Petterson B, Leonard H. Survival of infants born with Down's syndrome: 1980-96. Paediatr Perinat Epidemiol. 2000;14:163-71. Medline:10791661 doi:10.1046/j.1365-3016.2000.00252.x

12 Borenstein M, Hedges LV, Higgins JPT, Rothstein HR. Introduction to meta-analysis. Chichester, U.K.: John Wiley \& Sons; 2009.

13 DerSimonian R, Laird N. Metaanalysis in Clinical-Trials. Control Clin Trials. 1986;7:177-88. Medline:3802833 doi:10.1016/0197-2456(86)90046-2

14 Fjaerli HO, Farstad T, Bratlid D. Hospitalisations for respiratory syncytial virus bronchiolitis in Akershus, Norway, $1993-$ 2000: a population-based retrospective study. BMC Pediatr. 2004;4:25. Medline:15606912 doi:10.1186/1471-2431-4-25

15 Kristensen K, Hjuler T, Ravn H, Simoes EA, Stensballe LG. Chronic diseases, chromosomal abnormalities, and congenital malformations as risk factors for respiratory syncytial virus hospitalization: a population-based cohort study. Clin Infect Dis. 2012;54:810-7. Medline:22247121 doi:10.1093/cid/cir928

16 Stagliano DR, Nylund CM, Eide MB, Eberly MD. Children with Down syndrome are high-risk for severe respiratory syncytial virus disease. J Pediatr. 2015;166:703-9.e2. Medline:25596098 doi:10.1016/j.jpeds.2014.11.058

17 Zachariah P, Ruttenber M, Simoes EA. Down syndrome and hospitalizations due to respiratory syncytial virus: a population-based study. J Pediatr. 2012;160:827-31.e1. Medline:22177993 doi:10.1016/j.jpeds.2011.11.004

18 Simoes EA. Environmental and demographic risk factors for respiratory syncytial virus lower respiratory tract disease. J Pediatr. 2003;143(5 Suppl):S118-26. Medline:14615710 doi:10.1067/S0022-3476(03)00511-0

19 Duppenthaler A, Ammann RA, Gorgievski-Hrisoho M, Pfammatter JP, Aebi C. Low incidence of respiratory syncytial virus hospitalisations in haemodynamically significant congenital heart disease. Arch Dis Child. 2004;89:961-5. Medline:15383442 doi:10.1136/adc.2003.046714

20 Sommer C, Resch B, Simoes EA. Risk Factors for Severe Respiratory Syncytial Virus Lower Respiratory Tract Infection. Open Microbiol J. 2011;5:144-54. Medline:22262987 doi:10.2174/1874285801105010144

21 Boyce TG, Mellen BG, Mitchel EF Jr, Wright PF, Griffin MR. Rates of hospitalization for respiratory syncytial virus infection among children in medicaid. J Pediatr. 2000;137:865-70. Medline:11113845 doi:10.1067/mpd.2000.110531

22 Bertrand P, Navarro H, Caussade S, Holmgren N, Sánchez I. Airway anomalies in children with Down syndrome: endoscopic findings. Pediatr Pulmonol. 2003;36:137-41. Medline:12833493 doi:10.1002/ppul.10332

23 Uong EC, McDonough JM, Tayag-Kier CE, Zhao H, Haselgrove J, Mahboubi S, et al. Magnetic resonance imaging of the upper airway in children with Down syndrome. Am J Respir Crit Care Med. 2001;163:731-6. Medline:11254532 doi:10.1164/ajrccm.163.3.2004231

24 Joshi AY, Abraham RS, Snyder MR, Boyce TG. Immune evaluation and vaccine responses in Down syndrome: evidence of immunodeficiency? Vaccine. 2011;29:5040-6. Medline:21596078 doi:10.1016/j.vaccine.2011.04.060

25 de Hingh YC, van der Vossen PW, Gemen EF, Mulder AB, Hop WC, Brus F, et al. Intrinsic abnormalities of lymphocyte counts in children with down syndrome. J Pediatr. 2005;147:744-7. Medline:16356423 doi:10.1016/j.jpeds.2005.07.022

26 Cuadrado E, Barrena MJ. Immune dysfunction in Down's syndrome: primary immune deficiency or early senescence of the immune system? Clin Immunol Immunopathol. 1996;78:209-14. Medline:8605695 doi:10.1006/clin.1996.0031

27 Bloemers BL, Bont L, de Weger RA, Otto SA, Borghans JA, Tesselaar K. Decreased thymic output accounts for decreased naive T-cell numbers in children with Down syndrome. J Immunol. 2011;186:4500-7. doi:10.4049/jimmunol.1001700. Medline:21346234 
28 Bull MJ. Health supervision for children with Down syndrome. Pediatrics. 2011;128:393-406. doi:10.1542/peds.20111605. Medline:21788214

29 Weijerman ME, van Furth AM, Vonk Noordegraaf A, van Wouwe JP, Broers CJ, Gemke RJ. Prevalence, neonatal characteristic, and first-year mortality of Down syndrome: a national study. J Pediatr. 2008;152:15-9. doi:10.1016/j. jpeds.2007.09.045. Medline:18154890

30 Kim MA, Lee YS, Yee NH, Choi JS, Choi JY, Seo K. Prevalence of congenital heart defects associated with Down syndrome in Korea. J Korean Med Sci. 2014;29:1544-9. doi:10.3346/jkms.2014.29.11.1544. Medline:25408587

31 Joint Formulary Committee. British National Formulary. 72 ed. London: BMJ Group and Pharmaceutical Press; 2016. Section 5.3.5 Respiratory Syncytial Virus.

32 Yi H, Lanctôt KL, Bont L, Bloemers BL, Weijerman M, Broers C, et al; CARESS investigators. Respiratory syncytial virus prophylaxis in Down syndrome: a prospective cohort study. Pediatrics. 2014;133:1031-7. Medline:24799541 doi:10.1542/ peds.2013-3916

33 American Academy of Pediatrics Committee on Infectious Diseases; American Academy of Pediatrics Bronchiolitis Guidelines Committee. Updated guidance for palivizumab prophylaxis among infants and young children at increased risk of hospitalization for respiratory syncytial virus infection. Pediatrics. 2014;134:415-20. Medline:25070315 doi:10.1542/ peds.2014-1665

34 Bollani L, Baraldi E, Chirico G, Dotta A, Lanari M, Del Vecchio A, et al; Italian Society of Neonatology. Revised recommendations concerning palivizumab prophylaxis for respiratory syncytial virus (RSV). Ital J Pediatr. 2015;41:97. Medline:26670908 doi:10.1186/s13052-015-0203-x

35 Robinson JL, Le Saux N; Canadian Paediatric Society, Infectious Diseases and Immunization Committee. Preventing hospitalizations for respiratory syncytial virus infection. Paediatr Child Health. 2015;20:321-33. Medline:26435673

36 Megged O, Schlesinger Y. Down syndrome and respiratory syncytial virus infection. Pediatr Infect Dis J. 2010;29:672-3. doi:10.1097/INF.0b013e3181d7ffa5. Medline:20228692

37 National Institute for Health and Care Excellence (NICE). Bronchiolitis in children: diagnosis and management. NICE guideline (NG9) [Internet]. NICE:London;2015 June [cited 2017 May 26]. Available from: https://www.nice.org.uk/ guidance/ng9.

38 Ralston SL, Lieberthal AS, Meissner HC, Alverson BK, Baley JE, Gadomski AM, et al. Clinical practice guideline: The diagnosis, management, and prevention of bronchiolitis. Pediatrics. 2014;134:e1474-e1502. doi:10.1542/peds.2014-2742. Medline:25349312

39 Weber MW, Mulholland EK, Greenwood BM. Respiratory syncytial virus infection in tropical and developing countries. Trop Med Int Health. 1998;3:268-80. Medline:9623927 doi:10.1046/j.1365-3156.1998.00213.x

40 American Academy of Pediatrics Committee on Infectious Diseases and Committee on Fetus and Newborn. Revised indications for the use of palivizumab and respiratory syncytial virus immune globulin intravenous for the prevention of respiratory syncytial virus infections. Pediatrics. 2003;112:1442-6. Medline:14654627 doi:10.1542/peds.112.6.1442

41 Thiagarajan RR, Laussen PC. Mortality as an outcome measure following cardiac surgery for congenital heart disease in the current era. Paediatr Anaesth. 2011;21:604-8. doi:10.1111/j.1460-9592.2011.03580.x. Medline:21481081 